\title{
OCT of Optic Nerve Head in High Risk Group of Primary Open Angle Glaucoma
}

O.M.Kamal, H.M.Fayek and M.A.Elsayed

Ophthalmology Dept., Faculty of Medicine, Benha Univ., Benha, Egypt

E-Mail:mohammed2356@gmail.com

\begin{abstract}
Glaucoma is an optic neuropathy characterized by irreversible loss of neural tissue over time, Advancement in OCT technology has provided an objective and quantitative method to evaluate RNFL and optic nerve head which is clearly advantageous in the effective management of patients, both in terms of diagnosis and monitoring of response to therapy. to discuss the most important glaucoma-related applications of OCT A total of 48 eyes of participants were classified into 4 groups: patient with Family history of Primary open angle glaucoma(11 eyes), patients with Ocular hyper tension (13eyes), patients with black race (12eyes) and patients with high myopia (12eyes), All study subjects will undergo complete ophthalmic examination, clinical evaluation of optic disc and Spectral domain OCT system. the mean \pm SD age of the studied group was $52.2 \pm 20.2$ and $66.7 \%$ of them were males while $33.3 \%$ of them were females. Glaucoma was found in $62.5 \%$ of the examined high risk group while $37.5 \%$ of them were not glaucomatous, a statistically significant difference is found only in average RNFL thickness between normal group and glaucomatous group.
\end{abstract}

Keywords: OCT, Optic Nerve Head, Open Angle Glaucoma.

\section{Introduction}

Glaucoma is a chronic, progressive optic neuropathy with a specific pattern of structural and functional damages, And the leading cause of irreversible blindness worldwide. It is considered as a major public health concern, and its prevalence will continue [1].

Early diagnosis and appropriate treatment can slow the disease progression and preserve useful vision. The ability to diagnose glaucoma early and detect its progression sensitively is therefore very important for disease management, Optical coherence tomography (OCT) has been widely used in ophthalmology over the past 2 decades [2].

In clinical practice, OCT allows in vivo quantitative assessment of the per papillary retinal nerve fiber layer (RNFL) and the optic nerve head $(\mathrm{ONH})$ parameters with precision and good reproducibility, which is proved to be particularly valuable in glaucoma detection, staging, and monitoring [3].

Ocular hypertension is an established risk factor for glaucoma; Other risk factors for glaucoma prevalence include first-degree relatives with glaucoma, black race, and high myopia. The human retina contains more than 1 million retinal ganglion cells (RGCs), approximately $50 \%$ of which are concentrated in the foveal center [4].

Previous studies have confirmed that structural changes of glaucoma primarily affect RGC and their axons [5].

Theoretically, it is easier to detect the loss of RGC counts in the macula because of the high density in this region. The development of spectral-domain OCT (SDOCT) enables the measurement of macular ganglion cell complex (GCC) thickness. Defined as the sum of RNFL, ganglion cell layer (GCL), and inner plexiform layer (IPL) thickness, GCC was proved by several studies that it had a similar glaucoma discriminating performance with RNFL [6].

The purpose of this study was to highlighten the value of OCT in the early detection of glaucomatous damage in high risk group of primary open angle glaucoma .

\section{Patients and methods}

This study was an observational case control study, It was carried out between April 2019 and December 2019 in the Ophthalmology Department, Faculty of Medicine, Benha University Hospital. A total of 48 eyes of participants classified into 4 groups: patient with Family history of Primary open angle glaucoma(11 eyes), patients with Ocular hyper tension (13eyes), patients with black race (12eyes) and patients with high myopia (12eyes).

\section{The inclusion criteria}

- Age of participants : $20<$ Age $<80$ years.

- Best-corrected visual acuity of $6 / 24$ or better.

- patient with Family history of Primary open angle glaucoma.

or Patient with ocular hyper tention

or Patient with black race.

or Patient with high myopia.

- Both sexes

- Cooperative patient.

- Open ant.chamber angle.

The exclusion criteria

1)Patients under glaucoma medications.

2)Patients with Amblyopia.

3) Complicated intraocular surgery.

4)Secondary glaucoma (e.g. traumatic or inflammatory.

5)co-existing retinal diseases

6) Other diseases affecting visual field (e.g. pituitary lesion.

7)Corneal or lens opacity that interfere with clinical evaluation of the optic disc and posterior pole.

All study subjects will undergo complete ophthalmic examination including : Medical, ocular, and family histories (1st degree relative); Visual acuity (VA) testing by snellen chart; Intraocular pressure measurements by Goldmann's applanation tonometry; 
Gonioscopy for angle examination; Fundus examination.

Clinical evaluation of the optic disc was done by stereoscopic ophthalmoscopy with a hand-held 90-D lens.

Investigations were done in Ophthalmic Diagnostic and Laser Unit in Benha Hospital university by using Spectral domain OCT system (RTVueOCT; Optovue Inc., Fremont, CA, USA, software version $2017,1,0,151)$ of $840-n m$ wavelength was used to scan the optic disc and macula in all eyes. Peripapillary NFL thickness was measured from this traditional optic nerve head scan. This scan consists of 12 radial scans of $3.4 \mathrm{~mm}$ in length and 6 concentric ring scans all centered on the optic disc.

The ganglion cell complex (GCC) scan was done in all eyes with a square grid on the central macula and centered $1 \mathrm{~mm}$ temporal to the fovea. The GCC thickness was measured from the inner limiting membrane (ILM) to the posterior boundary of the inner plexiform layer. Mean, superior, and inferior GCC thicknesses were acquired.

\subsection{Statistical analysis}

The collected data were coded, entered, presented, and analyzed by computer using a data base software program, Statistical Package for Social Science (SPSS) version 16. Qualitative data were represented as frequencies and percent. For quantitative variables mean and standard deviation were computed and median computed in case of data not normally distributed. Chi square $\left(\mathrm{X}^{2}\right)$ tests were used to detect relation between different qualitative variables. Independent $\mathrm{T}$ test was used to calculate difference between quantitative variables in two groups in normally distributed data. Mann Whitney test was used to calculate difference between quantitative variables in two groups in not normally distributed data. The significance Level for all above mentioned statistical tests done, the threshold of significance is fixed at $5 \%$ level (P-value).

\section{Results}

This study was conducted on 48 eyes from patients of high risk for primary open angle glaucoma using OCT at the Ophthalmology Departments, Faculty of Medicine, Benha University Hospital, the mean \pm SD age of the studied group was $52.2 \pm 20.2$ and $66.7 \%$ of them were males while $33.3 \%$ of them were females.

Table (1) Age and sex distribution of the studied high risk group.

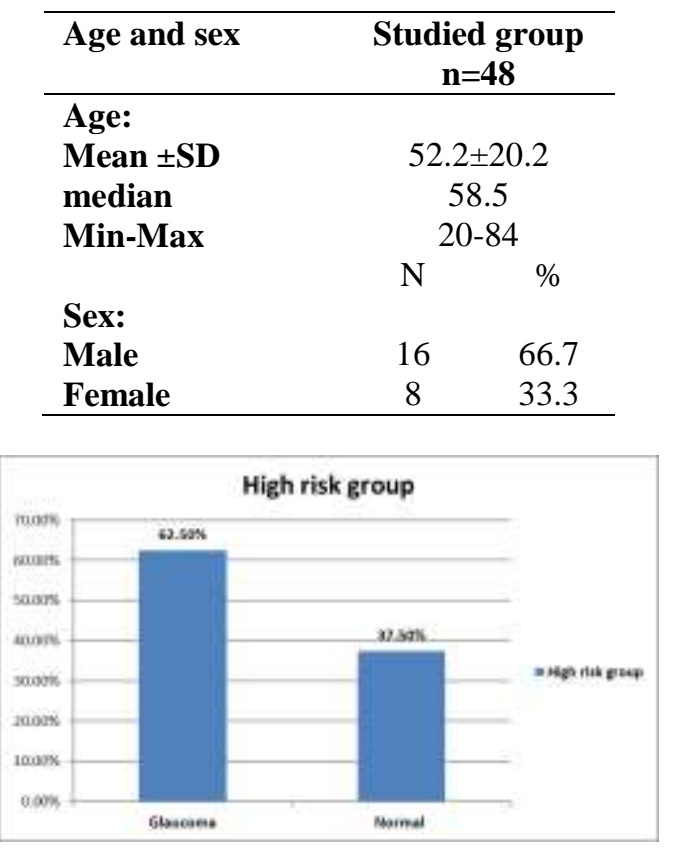

Fig (1) Frequency distribution of the studied high risk group regarding diagnosis of glaucoma.

Glaucoma was found in $62.5 \%$ of the examined high risk group while $37.5 \%$ of them were not glaucomatous.

Table (2) Relation between high risk factors in normal and glaucomatous group.

\begin{tabular}{|c|c|c|c|c|c|c|c|}
\hline \multirow[t]{2}{*}{$\begin{array}{l}\text { High Risk } \\
\text { factors }\end{array}$} & \multicolumn{2}{|c|}{$\begin{array}{c}\text { Glaucomatousgroup } \\
(\mathrm{n}=\mathbf{3 0})\end{array}$} & \multicolumn{2}{|c|}{$\begin{array}{c}\text { Non Glaucomatous } \\
\text { group }(n=18)\end{array}$} & \multirow[t]{2}{*}{ Total } & \multirow[t]{2}{*}{$X^{2}$} & \multirow[t]{2}{*}{ P-value } \\
\hline & $\mathbf{N}$ & $\%$ & $\mathbf{N}$ & $\%$ & & & \\
\hline Family history & 5 & 45.5 & 6 & $5 \leqslant .5$ & 11 & & \\
\hline $\begin{array}{l}\text { Ocular } \\
\text { hypertension }\end{array}$ & 10 & 76.9 & 3 & 23.1 & 13 & 4.1 & 0.25 \\
\hline Black race & 9 & 75.0 & 3 & 25.0 & 12 & & \\
\hline Myopia & 6 & 50.0 & 6 & 50.0 & 12 & & \\
\hline
\end{tabular}

This table shows that $76.9 \%$ of studied persons with ocular hypertension were diagnosed to have glaucoma and $75 \%$ of black race persons had glaucoma and $45.5 \%$ of family history persons had glaucoma and $50 \%$ of myopic patients had glaucoma. 
Table (3) Relation between IOP and BCVA in normal and glaucomatous group.

\begin{tabular}{|c|c|c|c|c|}
\hline IOP and BCVA & $\begin{array}{c}\text { Glaucomatousgroup } \\
(\mathbf{n}=30)\end{array}$ & $\begin{array}{l}\text { Non Glaucomatous } \\
\text { group }(n=18)\end{array}$ & Test & p-value \\
\hline IOP(mmhg): & \multirow{3}{*}{$\begin{array}{c}24.67 \pm 1.58 \\
22-27\end{array}$} & \multirow{3}{*}{$\begin{array}{c}15.9 \pm 3.68 \\
12-26\end{array}$} & & \\
\hline Mean \pm SD & & & $11.3^{\mathrm{a}}$ & $<0.001 * *$ \\
\hline \multirow{2}{*}{ Min-Max } & & & & \\
\hline & $\mathrm{N} \quad \%$ & $\mathrm{~N} \quad \%$ & & \\
\hline Normal IOP level $(n=15)$ & 0.0 & 100.0 & $36.3^{\mathrm{b}}$ & $<0.001 * *$ \\
\hline High IOP level $(n=33)$ & 90.9 & 9.1 & & \\
\hline BCVA: & \multirow{3}{*}{$\begin{array}{c}0.46 \pm 0.12 \\
0.2-0.7\end{array}$} & & & \\
\hline Mean \pm SD & & \multirow{2}{*}{$\begin{array}{c}0.96 \pm 0.09 \\
0.7-1.0\end{array}$} & $15.1^{\mathrm{a}}$ & $<0.001 * *$ \\
\hline \multirow[t]{2}{*}{ Min-Max } & & & & \\
\hline & $\%$ & $\%$ & & \\
\hline$\leq \operatorname{median}(0.6)(n=29)$ & 100.0 & 0.0 & $43.9^{b}$ & $<0.001 * *$ \\
\hline$>\operatorname{median}(0.6)(n=19)$ & 5.3 & 94.7 & & \\
\hline
\end{tabular}

${ }^{\mathrm{a}}=\mathrm{t}$-test ${ }^{\mathrm{b}}=$ chi square test $\quad$ BCVA: best corrected visual acuity

** High statistical significant difference $(\mathrm{P}<0.001)$.

The mean \pm SD of IOP in glaucomatous group was highly statistical significant $(\mathrm{p}<0.001)$ higher than that of non glaucomatous group $(24.67 \pm 1.58$ and $15.9 \pm 3.68$ respectively).

Also, $90.9 \%$ of studied persons with high IOP were diagnosed to have glaucoma and this relation of high IOP and glaucoma was highly significant $(\mathrm{p}<0.001)$. Moreover, the mean \pm SD of BCVA in glaucomatous group was highly statistical significant $(\mathrm{p}<0.001)$ lower than that of non glaucomatous group $(0.46 \pm 0.12$ and $0.96 \pm 0.09$ respectively), also $100 \%$ of persons who had $\mathrm{BCVA}<0.6$ were diagnosed as glaucoma and this relation of lower BCVA and glaucoma was highly significant $(\mathrm{p}<0.001)$.

Table (4) Relation between retinal nerve fiber layer (RNFL) in normal and glaucomatous group.

\begin{tabular}{|c|c|c|c|c|}
\hline RNFL & $\begin{array}{c}\text { Glaucomatousgroup } \\
(\mathrm{n}=\mathbf{3 0})\end{array}$ & $\begin{array}{l}\text { Non Glaucomatous } \\
\text { group }(n=18)\end{array}$ & Test & p-value \\
\hline Average RNFL( $\mu \mathrm{m})$ : & \multirow{3}{*}{$\begin{array}{c}72.2 \pm 12.5 \\
41-93\end{array}$} & \multirow{3}{*}{$\begin{array}{c}95.7 \pm 8.5 \\
83-113\end{array}$} & & \\
\hline Mean \pm SD & & & $7.1^{\mathrm{a}}$ & $<0.001 * *$ \\
\hline \multirow[t]{2}{*}{ Min-Max } & & & & \\
\hline & $\mathrm{N} \quad \%$ & $\mathrm{~N} \quad \%$ & & \\
\hline$\leq \operatorname{median}(81)(n=25)$ & 100.0 & 0.0 & $1.3^{\mathrm{b}}$ & $<0.001 * *$ \\
\hline$>\operatorname{median}(81)(n=23)$ & 21.7 & 28.3 & & \\
\hline \multicolumn{5}{|l|}{ Superior RNFL $(\mu \mathrm{m})$ : } \\
\hline Mean \pm SD & $73.3 \pm 12.8$ & $97.06 \pm 10.67$ & $6.6^{\mathrm{a}}$ & $<0.001 * *$ \\
\hline \multirow[t]{2}{*}{ Min-Max } & $46-96$ & 83-121 & & \\
\hline & $\%$ & $\mathrm{~N} \quad \%$ & & \\
\hline$\leq \operatorname{median}(84.5)(n=24)$ & 95.8 & 4.2 & $22.7^{\mathrm{b}}$ & $<0.001 * *$ \\
\hline$>\operatorname{median}(84.5)(n=24)$ & 29.2 & 70.8 & & \\
\hline \multicolumn{5}{|l|}{ Inferior RNFL $(\mu \mathrm{m})$ : } \\
\hline Mean \pm SD & \multirow{2}{*}{$\begin{array}{c}70.17 \pm 15.02 \\
36-101\end{array}$} & \multirow{2}{*}{$\begin{array}{c}94.06 \pm 7.46 \\
83-110\end{array}$} & $6.3^{\mathrm{a}}$ & $<0.001 * *$ \\
\hline \multirow[t]{2}{*}{ Min-Max } & & & & \\
\hline & $\%$ & $\mathrm{~N} \quad \%$ & $28.8^{\mathrm{b}}$ & \\
\hline$\leq \operatorname{median}(82)(n=24)$ & 100.0 & 0.0 & & $<0.001 * *$ \\
\hline$>\operatorname{median}(82)(n=24)$ & 25.0 & 75.0 & & \\
\hline
\end{tabular}

The mean \pm SD of average, superior and inferior RNFL thickness in glaucomatous group was highly statistical significant $(\mathrm{p}<0.001)$ lower than that of non glaucomatous group. Also there was high statistical significant relation $(\mathrm{p}<0.001)$ between low nerve fiber thickness and glaucoma as $100 \%$ of persons with average RNFL thickness $\leq 81 \mu \mathrm{m}, 95.8 \%$ of persons with superior RNFL thickness $\leq 84.5 \mu \mathrm{m}$ and $100 \%$ of persons with inferior RNFL thickness $\leq 82 \mu \mathrm{m}$ were diagnosed as glaucoma. 
Table (5) Relation between cup-to- disc ratio in normal and glaucomatous group.

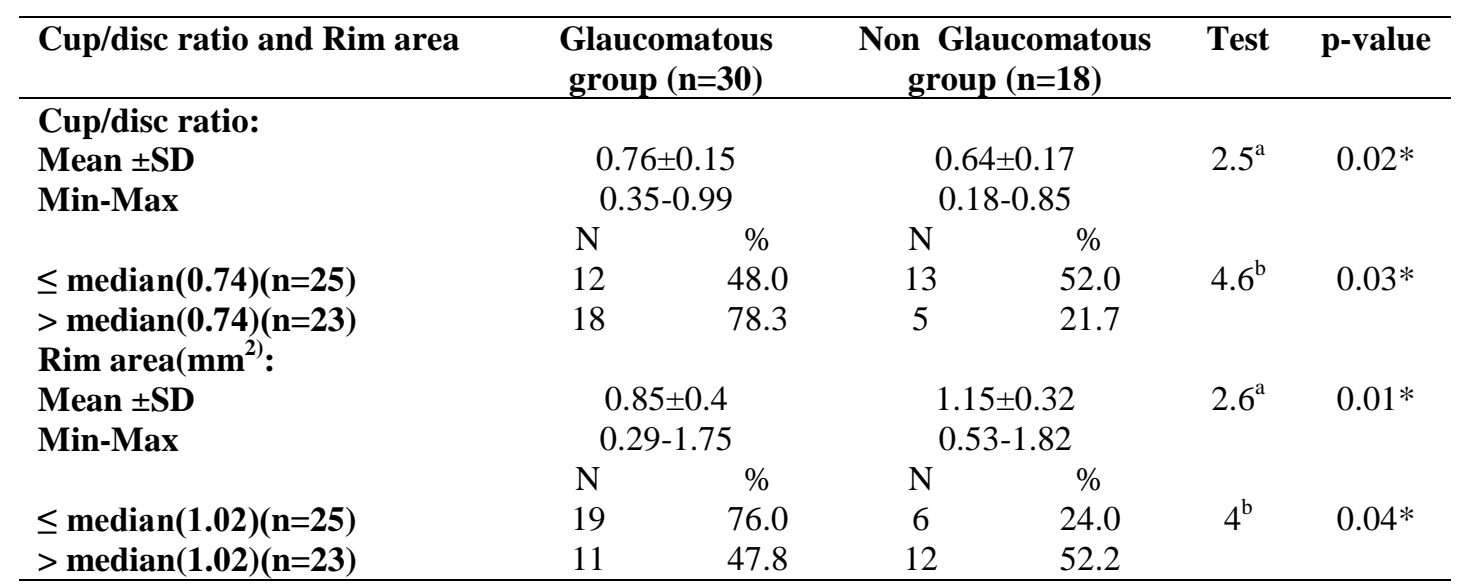

$$
{ }^{a}=\text { t-test }^{b}=\text { chi square test } \quad * * \text { High statistical significant difference }(P<0.001)
$$

The mean \pm SDcup/disc ratio in glaucomatous group was statistically significant $(\mathrm{p}<0.05)$ higher than that of non glaucomatous group $(0.76 \pm 0.15$ vs. $0.64 \pm 0.17)$. Also there was statistical significant relation $(\mathrm{p}<0.05)$ between increased cup/disc ratio $>0.74$ and glaucoma as $78.3 \%$ of persons with increased Cup/disc ratio had

\section{Discussion}

Regarding the role of macular thickness parameters in detecting glaucoma has been previously reported [7], as ganglion cells are thickest at the perifoveal area and constitute around $30 \%-35 \%$ of retinal thickness in this region. GCC encompasses three layers in the retina, comprising the retinal nerve fiber layer (NFL), ganglion cell layer (GCL), and inner-plexiform layer (IPL). Both NFL and GCL become thinner as ganglion cells die from glaucoma [7]. Measurements of the GCC, proving the death of ganglion cells, definitely improved discriminatory ability [8].

In this study, average GCC, Superior GCC, and inferior GCC thickness measurement were lower in the glaucomatous group, but showed no statistically significant differences between both groups ( $p>0.05)$, Another GCC parameters showed different results.

These results were partly consistent with those obtained by Shoji et al. Their study assessed the glaucomatous changes in high myopes using the SDOCT and concluded that GLV and FLV showed a good detectability of glaucomatous changes in high myopes, but the difference from our study is that they found that FLV and GLV had nearly the same detectability as average GCC [7]. This is opposite to our study where we found no significant ability in detecting glaucoma in high myopes using the average GCC thickness measurement.

Other studies have also found that the GCC analysis in the RTVue-OCT had an advantage over the other parameters for diagnosing glaucoma in high myopia, like the one done by Shoji et al. [7].The study glaucoma. Regarding the rim area thickness it was statistically lower $(\mathrm{p}<0.05)$ in glaucomatous group than non glaucomatous group $(0.85 \pm 0.4$ and $1.15 \pm 0.32$ respectively) and the relation was significant $(\mathrm{p}<0.05)$ between low thickness $<1.02 \mathrm{~mm} 2$ and glaucoma as $76 \%$ of persons with low rim thickness had a glaucoma

enrolled 51 perimetric glaucomatous eyes with high myopia and 31 highly myopic eyes without glaucomatous visual field loss.

They reported that macular GCC measurements were significantly better than cpRNFL measurements at detecting perimetric glaucoma in high myopia cohorts.

Notably, Shoji et al [7] showed that the diagnostic accuracy of macular GCC does not decrease in highly myopic patients, unlike peripapillary RNFL thickness.

In a following study, they added an emmetropic comparison group, and showed that only cpRNFL measurement had a decreased ability to detect glaucoma in the High Myopic Glaucoma group (HMG), whereas macular GCC measurements efficiently detected glaucoma in both the HMG and the Emmetropic Glaucomatous groups.

On the other hand, 2 studies were found to have different conclusions than the above mentioned opinions. Choi et al [9] compared the glaucoma detection ability of macular ganglion cell-inner plexiform layer (GCIPL) thickness measured with Cirrus spectral-domain optical coherence tomography (SD-OCT) with that of peripapillary retinal nerve fiber layer (RNFL) thickness in high myopia. They concluded that the glaucoma detection ability of macular thickness was high and comparable with that of peripapillary RNFL thickness in high myopes. The other study by Kim et al. [10] was done to compare the diagnostic ability to detect glaucomatous changes between the macular ganglion cell complex (GCC) and peripapillary retinal nerve fiber layer (RNFL) thickness in highly myopic patients using Fourier-Domain OCT, They found that the ability to diagnose glaucoma with 
macular GCC thickness was comparable to that with peripapillary RNFL thickness in high-myopia patients.

As macular GCC thickness is proved to be less affected by axial length than peripapillary RNFL thickness, they suggested that macular GCC thickness measurements may be a good alternative or a complimentary measurement to RNFL thickness assessment in the clinical evaluation of glaucoma in patients with high myopia. Two reasons for the superiority of the macular parameters have been proposed: Firstly, many studies showed that the retinal thinning in myopia was found mainly in the peripheral areas, and that the retinal thickness in the central area is preserved or even thicker in high myopia. The thinning of the GCC and GCIPL thicknesses are supposed to be due to glaucomatous RGC loss, rather than high myopia, since the central areas are less influenced by elongated axial lengths. Secondly, the diameters of the scan circles used in the optic disc protocols are fixed, and the peripapillary disc margins are not that reliable, since most of the optic discs in highly myopic eyes displayed tilted, torsional, and peripapillary atrophic appearances [11].

The current study has its points of strength as well as limitations. The study acquire strength from comparing glaucoma patient to non glaucoma patients sharing the same condition which is myopia, and avoiding inclusion of non-myopic subjects which may over-estimate the performance of the machine, as suggested by Medieros [3]. In addition, one eye from each subject was included in the study avoiding "both eyes" source of bias. The limitation to the study is related to the OCT technology and its lack of accuracy and reproducibility in very high error of myopia due to presence of posterior staphyloma and long axial length, which led to exclusion of high degrees of myopia from inclusion in the study. A longitudinal study could benefit in detecting the changes occurring in OCT parameters in myopic glaucoma patients over time.

Regarding ocular hypertention; In our study, significant differences between groups are set in all ONH parameters, except optic disc area. Rim area was established as the ONH parameter with the highest differences between groups and it was observed as the only $\mathrm{ONH}$ parameter with a significant difference between late stage POAG group and other groups.

This finding shows that rim area decreases to an important degree in late stage glaucoma patients. Wollstein et al [12] report that the most valuable $\mathrm{ONH}$ parameter in differentiating POAG patients from normal group handled with OCT is rim area.

Correspondingly, Aydogan et al [13] recognize rim area as the $\mathrm{ONH}$ parameter showing the highest correlation with RNFL thickness in their study with SD-OCT (Cirrius), and emphasize that rim area is the most valuable parameter among $\mathrm{ONH}$ data in diagnosis.

In our results, showing the differences between groups that had already expected with OCT could be important to attract attention to this new imaging method in glaucoma.
Schuman et al [14] found RNFL thickness in their study with stratus OCT as $95.9 \pm 10.09 \mu \mathrm{m}$ in the normal group, $80.3 \pm 18.4 \mu \mathrm{m}$ in early stage glaucoma patients, and $50.7 \pm 13.6 \mu \mathrm{m}$ in late stage glaucoma patients respectively. They reported that RNFL thickness measured via OCT showed significant difference between healthy and glaucoma eyes.

In our study, average RNFL thicknesses handled with SD-OCT were found as $94.52 \pm 9.31 \mu \mathrm{m}$ in the normal group , 88.76 $\pm 14.87 \mu \mathrm{m}$ in the $\mathrm{OH}$ group, To assess RNFL thickness of quadrants handled with OCT is also important. Guedes et al ascertained that all RNFL parameters decrease significantly in glaucoma group, and only inferior RNFL thickness decreases in glaucoma suspected group in their study.

Leung et al [15] reported that RNFL thickness, especially in the inferior quadrant, showed significant difference between glaucoma suspected group and normal group in their study. They also showed that there were significant differences in all quadrants, except temporal quadrant in normal and glaucoma eye, Differences in RNFL thickness determined in our study are similar to results of previous studies. The most evident slimming is observed in the inferior quadrant, in accordance with the rule described as ISNT rule by some researchers, and shows the sequence of RNFL loss. Superior, nasal and temporal quadrants follow this . It has been determined that changes in inferior quadrant RNFL thickness is more important than other RNFL thickness data involving average RNFL thickness in the assessment and following of glaucoma patients.

In our study, a statistically significant difference is found only in average RNFL thickness between normal group and glaucomatous patients.

Lederer et al [16] detected that macular thickness was thinner in a statistically significantly way in early stage glaucoma cases than in normal cases. Also, they found macular thickness in late stage galucoma cases as normal, but significantly thinner than early stage galucoma group and glaucoma suspected cases. No difference in foveal thickness was seen in any group, and morphological changes in foveal region do not seem to be beneficial in the diagnosis and treatment of glaucoma patients. Another noteworthy point from our results is that there was no significant difference in any macular parameter detected via OCT between $\mathrm{OH}$ and healthy groups. Conversely, significant difference in all macular thickness data, except fovea, was observed between $\mathrm{OH}$ patients and POAG groups involving early stage glaucoma.

Ojima et al [17] reported a significant decrease in six of nine macula segments at early stage of glaucoma and normal foveal thickness even at late stages. When POAG patients are compared with the normal group, an increase in the difference between foveal thickness and peripheral macular thickness going away from center of macula is evident. Macular ganglion cell asymmetry analysis showed good glaucoma diagnostic ability, especially in early-stage glaucoma [18]. 


\section{Conclusion}

OCT presents valuable information that could direct doctors when diagnosing and treating glaucoma, by providing objective and reliable data regarding peripapiler nerve fibar layer thickness, macular thickness measurement and optic disc parameters. Although assessment of $\mathrm{ONH}$ and the analysis of macular thickness (a relatively new method) are important in the diagnosis and treatment of glaucoma, the assessment of RNFL still seems to be the most valuable parameter. The diagnostic value of macular thickness analysis following RNFL assessment is noteworthy, but the assessment of OCT data, together with clinical findings, is of critical importance in the assessment of high risk glaucoma patients. In addition, a high inter visit reproducibility of the SD-OCT parameters, which is very useful in monitoring disease progression and the course of treatment. The OCT parameter with the highest diagnostic value in POAG is the inferior RNFL thickness. Also, the parameter with the highest diagnostic value among $\mathrm{ONH}$ data is the rim region.

\section{References}

[1] C. Cook and P. Foster, "Epidemiology of glaucoma: what's new?," Can. J. Ophthalmol., Vol. 47 (3), PP. 223-226, 2012.

[2] X. Xu et al., "Diagnostic ability of macular ganglion cell-inner plexiform layer thickness in glaucoma suspects," Medicine (Baltimore)., Vol. 96(51), PP. 23-34, 2017.

[3] F. A. Medeiros, L. M. Zangwill, C. Bowd, R. M. Vessani, R. Susanna Jr, and R. N. Weinreb, "Evaluation of retinal nerve fiber layer, optic nerve head, and macular thickness measurements for glaucoma detection using optical coherence tomography," Am. J. Ophthalmol., Vol. 139(1]) PP. 44-55, 2005.

[4] M. Agudo-Barriuso, M. P. Villegas-Perez, J. M. de Imperial, and M. Vidal-Sanz, "Anatomical and functional damage in experimental glaucoma," Curr. Opin. Pharmacol., Vol. 13(1), PP. 5-11, 2013.

[5] F. A. Medeiros, R. Lisboa, R. N. Weinreb, J. M. Liebmann, C. Girkin, and L. M. Zangwill, "Retinal ganglion cell count estimates associated with early development of visual field defects in glaucoma," Ophthalmology, Vol. 120(4), PP. 736-744, 2013.

[6] N. R. Kim et al., "Comparing the ganglion cell complex and retinal nerve fibre layer measurements by Fourier domain OCT to detect glaucoma in high myopia," $\mathrm{Br}$. J. Ophthalmol., Vol. 95(8), PP. 1115-1121, 2011.

[7] T. Shoji, H. Sato, M. Ishida, M. Takeuchi, and E. Chihara, "Assessment of glaucomatous changes in subjects with high myopia using spectral domain optical coherence tomography," Invest. Ophthalmol. Vis. Sci., Vol. 52(2), PP. 1098-1102, 2011.

[8] H. Ishikawa, D. M. Stein, G. Wollstein, S. Beaton, J. G. Fujimoto, and J. S. Schuman, "Macular segmentation with optical coherence tomography," Invest. Ophthalmol. Vis. Sci., Vol. 46(6), PP. 2012 2017, 2005.

[9] Y. J. Choi, J. W. Jeoung, K. H. Park, and D. M. Kim, "Glaucoma detection ability of ganglion cellinner plexiform layer thickness by spectral-domain optical coherence tomography in high myopia," Invest. Ophthalmol. Vis. Sci., Vol. 54(3), PP. 22962304, 2013.

[10] J. H. Kim et al., "Prevalence and characteristics of glaucoma among Korean adults," Korean J. Ophthalmol., Vol. 25(2), PP. 110-115, 2011.

[11]M. C. C. Lim et al., "Use of optical coherence tomography to assess variations in macular retinal thickness in myopia," Invest. Ophthalmol. Vis. Sci., Vol. 46(3), PP. 974-978, 2005.

[12] G. Wollstein, H. Ishikawa, J. Wang, S. A. Beaton, and J. S. Schuman, "Comparison of three optical coherence tomography scanning areas for detection of glaucomatous damage," Am. J. Ophthalmol., Vol. 139(1), PP. 39-43, 2005.

[13] T. Aydoğan, B. İ. S. Akçay, E. Kardeş, and A. Ergin, "Evaluation of spectral domain optical coherence tomography parameters in ocular hypertension, preperimetric, and early glaucoma," Indian J. Ophthalmol., Vol. 65(11), p. 1143, 2017.

[14] J. S. Schuman et al., "Quantification of nerve fiber layer thickness in normal and glaucomatous eyes using optical coherence tomography: a pilot study," Arch. Ophthalmol., Vol. 113(5), PP. 586-596, 1995.

[15] C. K. S. Leung et al., "Comparison of macular and peripapillary measurements for the detection of glaucoma: an optical coherence tomography study," Ophthalmology, Vol. 112(3), PP. 391-400, 2005.

[16] D. E. Lederer et al., "Analysis of macular volume in normal and glaucomatous eyes using optical coherence tomography," Am. J. Ophthalmol., Vol. 135(6), PP. 838-843, 2003.

[17] T. Ojima, T. Tanabe, M. Hangai, S. Yu, S. Morishita, and N. Yoshimura, "Measurement of retinal nerve fiber layer thickness and macular volume for glaucoma detection using optical coherence tomography," Jpn. J. Ophthalmol., Vol. 51(3), PP. 197-203, 2007.

[18] Y. H. Hwang, S. Il Ahn, and S. J. Ko, "Diagnostic ability of macular ganglion cell asymmetry for glaucoma," Clin. Experiment. Ophthalmol., Vol. 43(8), PP. 720-726, 2015. 